F1-CN-64/D2-6

\title{
Recent Progress in Linear and Nonlinear Studies of Toroidal Alfvén Eigenmode
}

\author{
G. Y. Fu, Y. Chen, R. Budny, Z. Chang, C. Z. Cheng, D. S. Darrow,
} E. Fredrickson, E. Mazzucato, R. Nazikian, W. Park,

R. White, K. L. Wong, Y. Wu, S. Zweben

Princeton University Plasma Physics Laboratory, Princeton, New Jersey

D. A. Spong

Oak Ridge National Laboratory, Oak Ridge, Tennessee, USA

H. Kimura, T. Ozeki, and M. Saigusa

JAERI, Naka-machi, Naka-gun, Ibaraki-ken 311-01, Japan

M. S. Chu, W. W. Heidbrink and E. J. Strait

General Atomics, San Diego, California, USA

\section{DISCLAIMER}

This report was prepared as an account of work sponsored by an agency of the United States Government. Neither the United States Government nor any agency thereof, nor any of their employees, makes any warranty, express or implied, or assumes any legal liability or responsibility for the accuracy, completeness, or usefulness of any information, apparatus, product, or ence herein to any specific comts that its use would not infringe privately owned rights. Refermanufacturer, or otherwise does not product, process, or service by trade name, trademark, mendation, or favoring by thes not necessarily constitute or imply its endorsement, recomand opinions United States Government or any agency thereof. 


\section{DISCLAIMER}

Portions of this document may be illegible in electronic image products. Images are produced from the best available original document. 


\begin{abstract}
TAE modes are studied in linear and nonlinear regimes using several kinetic/MHD hybrid models. It is shown that the stability of TAE mode is largely determined by its radial mode structure. The calculated stability thresholds are correlated well with observations, including the recently observed alpha-driven TAE modes in the TFTR DT experiments. In the nonlinear regime, quasilinear simulations with multiple modes show that the saturation level is enhanced by nonlinear wave-particle resonance overlapping when the linear growth rate exceeds a critical value. A fully self-consistent $\delta$ f noise reduction method for the 3D particle/MHD hybrid model is developed.
\end{abstract}

\title{
1. Introduction
}

An important issue for tokamak fusion reactors is whether the Toroidal Alfvén Eigenmode[1] (TAE) can become unstable[2] and affect alpha particle confinement. This issue has been assessed in the past experiments where the TAE modes were strongly destabilized by energetic beam ions in the NBI-heated plasmas or by fast minority tail ions in the ICRF-heated plasmas[3-6]. Recently, alpha-driven TAE instability has been observed in the TFTR DT experiments[7] with reduced central magnetic shear and elevated central safety factor, although alpha particle loss has not yet been seen. In ITER, high-n TAE modes are predicted to be unstable. This gives new impetus to benchmark codes with experimental results and to study alpha particle loss induced by TAE modes. In this paper, recent results of linear and nonlinear studies of TAE modes are presented.

\section{Linear Stability}

The linear stability of TAE modes is studied using a kinetic MHD stability code NOVA-K $[8,9]$. The NOVA-K code is applied to several recent experiments, including the TFTR DT experiments, the JT-60U ICRF experiments and the DIII-D NBI experiments. The NOVA-K code calculates perturbatively the fast ion drive, the electron/ion/beam Landau damping, the collisional damping due to trapped electrons, and the "radiative" damping due to the coupling to the radially propagating kinetic Alfvén waves. The important physics of finite orbit width of fast ions is included. The continuum damping is neglected. All the calculations are valid for realistic equilibria with experimental parameters and profiles as obtained from the TRANSP code[10].

The TAE modes are calculated to be stable $[11,12]$ in the initial TFTR DT experiments in supershot regime with up to $10 \mathrm{MW}$ of fusion power, consistent with the observations[13]. The main damping mechanisms are beam ion Landau damping and the "radiative" damping. The stability is sensitive to the mode structure which depends on the details of plasma parameters and profiles, especially the $q$ profiles. There are two types of TAE modes as shown in Fig. 1: (a) the global modes and (b) the core-localized modes (CLM). The CLMs $[14,15]$ peak near the center of the plasma where the magnetic shear is weak, and tend to be less stable than the global modes since the alpha pressure profile is sharply peaked at the center. The stability of CLM is sensitive to the 

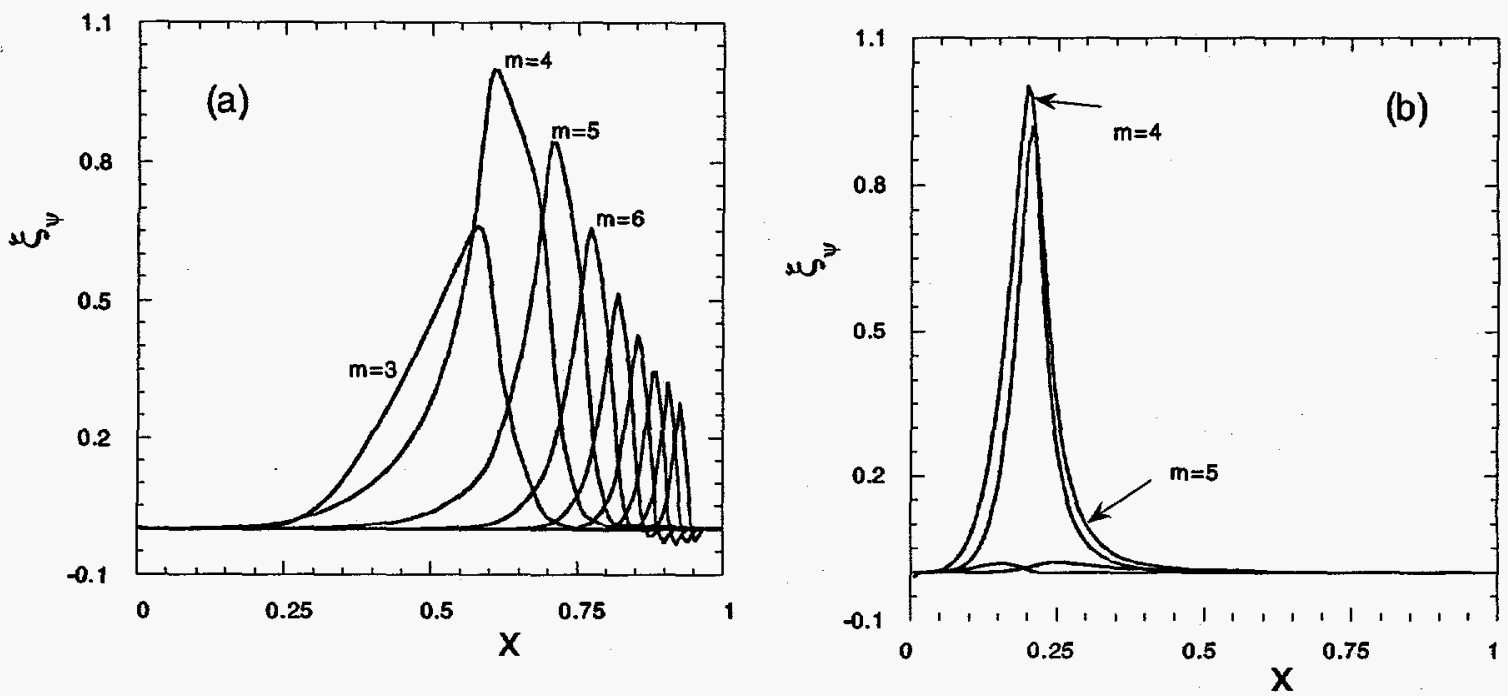

Figure 1: The radial mode structure of (a) a global mode and (b) a CLM mode.

central magnetic shear. Since the radial mode width is inversely proportional to the magnetic shear, a weaker shear results in larger alpha drive and smaller "radiative" damping. This led to the prediction[12] that the CLM modes can be destabilized by alpha particles with a weakly negative central magnetic shear and small beam ion Landau damping in the TFTR DT experiments. Figure 2 show the ratio of alpha drive to the total damping as a function of the magnetic shear at $r / a=0.15$ for a $n=5$ CLM in a high fusion power TFTR DT discharge (\#76770). We see that the mode becomes unstable as the shear becomes smaller. It should also be pointed out that the beam damping has an important effect on the stability. This implies that the mode tends to become more unstable when the beam ions slow down after the beam power is turned off. These results are confirmed qualitatively by the recent observation of alpha-driven TAE mode activity after NBI in the TFTR DT experiments[7].

In these new DT experiments, the plasmas have higher central safety factor $(q(0)>1)$, lower central magnetic shear and lower beta values. Figure 3 shows the calculated ratio of alpha drive to the damping of a $n=3$ mode versus $q(0)$ for such a plasma (TFTR shot \#93404) where the central alpha beta value is $0.014 \%$ (at $t=3.2 \mathrm{sec}$, when the instability appeared). The corresponding alpha beta threshold is very low, on order of $\beta_{\alpha}(0)=0.01 \%$ for the likely range of $2.3<q(0)<2.5$. This is in qualitative agreement with the experimental results. Physically, such low stability threshold is caused by several factors. First, the beam damping is significantly reduced due to slowing down of beam ions. Second, the plasma beta is reduced after NBI, which leads to smaller radiative damping, especially for the CLM modes. Finally, the alpha drive is relatively larger for higher $q(0)$ (when plasma beta is sufficiently low) and weaker magnetic shear. It can be shown analytically that the alpha drive is approximately proportional to $q / s$, where $s$ is the magnetic shear. From Fig. 3 , we also see that the stability is a sensitive function of $q(0)$. The instability is maximized at about $q(0)=2.45$ at which the CLM is located in the region of large alpha pressure gradient. As $q(0)$ varies from 2.5 to smaller value, the 


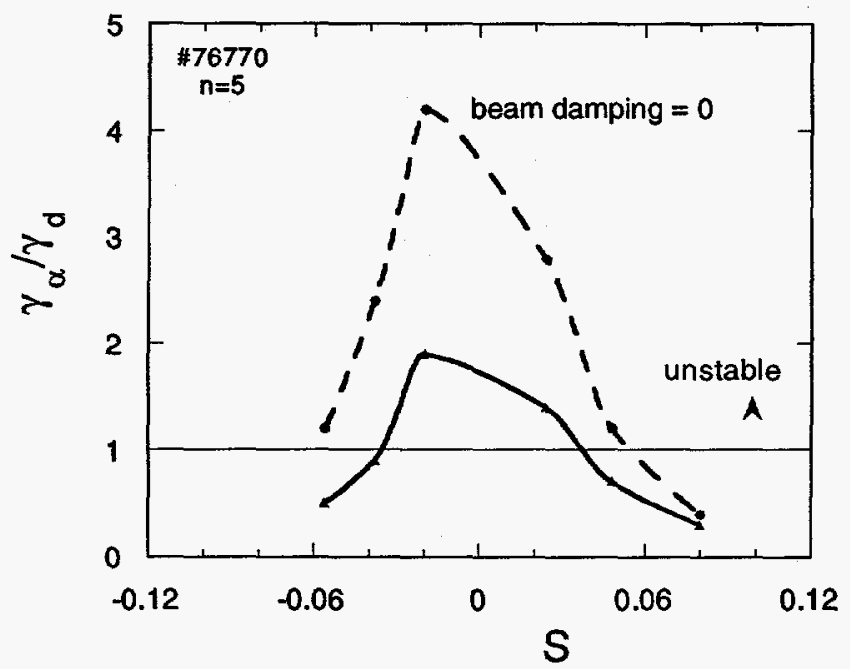

Figure 2: The ratio of alpha drive to the total damping versus the central magnetic shear with (dashed line) and without (solid line) beam damping.

mode location shifts from the center of the plasma toward the edge. This change in the mode location results in large variation of alpha particle drive. Work is in progress to analyze the alpha-driven TAE experiments in TFTR for a wider parameter range and results will be reported elsewhere.

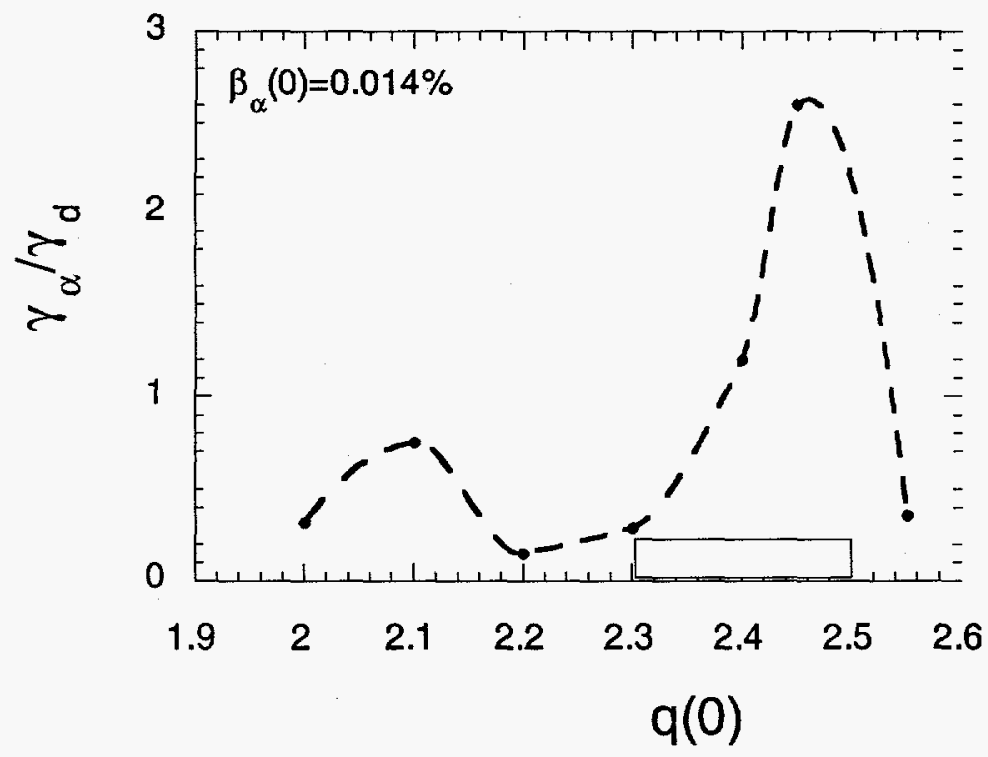

Figure 3: The ratio of drive to damping versus $q(0)$ for a $n=3$ mode in a TFTR DT discharge (\#93404).

The stability of TAE mode in the TFTR DT plasmas has also been analyzed using a gyrofluid model[16]. The model calculates excitation of the TAE using a set of fluid moment equations which have been constructed to include Landau resonance effects. The model also includes continuum damping, ion/electron 
Landau damping and the radiative damping. The fast ion distribution is currently constrained to be a Maxwellian. The calculations are performed with realistic equilibria and are non-perturbative. We find that the conventional global TAE and the CLM modes can be present for different parameter regimes. The parameter which determines which mode dominates is the ratio of the averaged alpha velocity to the Alfvén velocity $\left(v_{\alpha} / v_{A}\right)$. Figure 4 shows the linear growth rates as a function of $v_{\alpha} / v_{A}$ for the global mode and the CLM mode and several toroidal mode numbers. As indicated, the CLM mode resonates at somewhat lower values of $v_{\alpha} / v_{A}\left(0.3<v_{\alpha} / v_{A}<0.6\right)$ than the global TAE $\left(0.6<v_{\alpha} / v_{A}<1\right)$. Realistically, the alpha energy distribution and $v_{A}$ are constantly evolving in time so making a scan in $v_{\alpha} / v_{A}$ can be viewed as encompassing the dynamic behavior of the actual experiment.

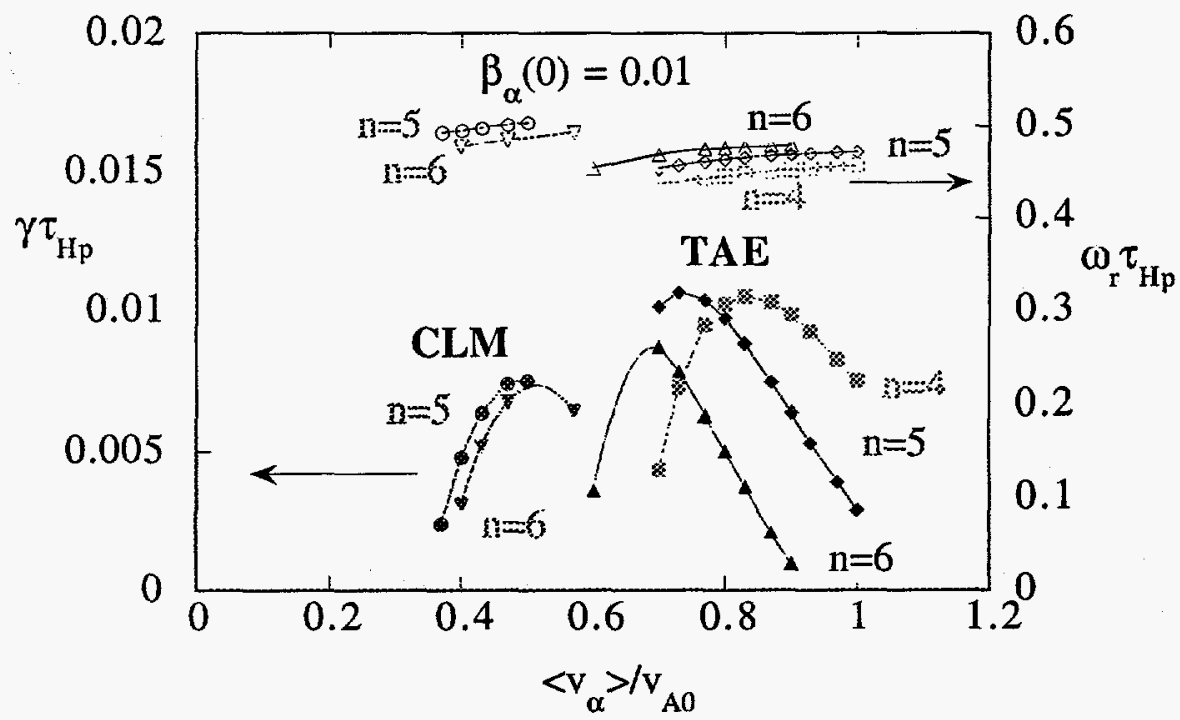

Figure 4: The growth rates and the real frequencies of the CLM mode and the global modes as a function of $v_{\alpha} / v_{A}$ for several toroidal mode numbers.

The stability of TAE in JT60U and DIII-D plasmas has also been analyzed using the NOVA-K code. In the recent JT-60U ICRF experiments, the high-n TAE modes were excited sequentially during the sawteeth stabilizing phases. The instability was induced by the fast minority ions powered by the ICRF heating. The results of NOVA-K code indicated that the stability of the TAE mode is a strong function of mode location[17]. This dependence results in sequential mode excitation as $q(0)$ drops in time. More recently, high-n TAE instability was observed in the JT-60U ICRF experiments with reversed shear profiles[18]. The mode activity only appeared after the second mini-collapse. Our calculations show that the stability is mainly determined by mode structure. It is found that that the mode is unstable only when it peaks in the region of weakly negative shear region just inside the $q_{\min }$ radius since the fast ion drive of such a mode is much larger than that of the mode which peaks outside the $q_{\min }$ radius. Furthermore, the existence of the mode just inside $q_{\min }$ depends on the density gradient in this region. The mode exist only when the density gradient is small or the continuum gaps are well aligned near the $q_{\min }$ radius. 
This result is in good agreement with the experimental observations since the mini-collapses reduce the density gradient significantly. Details of these results are reported by Kimura et al.[18] at this conference. Finally, for the DIII-D NBI experiments, the calculated critical beam ion beta is $\beta_{\text {beam }}(0) \approx 10 \%$ for the TAE instability. This is in approximate agreement with the observations. Compared to the TFTR NBI and the JT-60U ICRF experiments, the critical fast ion beta in the DIII-D NBI-heated plasmas is much higher due to the edge localized mode structure.

\section{Nonlinear Particle/MHD Hybrid Simulations}

Here, we study nonlinear evolution of the TAE modes using Particle/MHD hybrid simulations. Two models are used: a quasilinear model developed by Chen and White[19] and the fully nonlinear model by Park et al.[20]. First, the quasilinear model is used to study the saturation of TAE modes. The model is valid when the mode amplitudes are low enough such that the mode-mode coupling effects can be neglected in the nonlinear stage. In this limit, each mode is described by a slowly varying amplitude and phase with fixed mode structure. Given the linear mode structure $\eta_{n}(x)$, we can represent the solution as

$$
\xi(\mathrm{x}, t)=\sum A_{n}(t) \eta_{n}(\mathrm{x}) \sin \left(\omega_{n} t+\alpha_{n}(t)\right)
$$

where $\omega_{n}$ is the mode frequency. The equations for the amplitude $A_{n}(t)$ and the phase $\alpha_{n}(t)$ can be derived from the momentum equation with the currentcoupling scheme and are given by

$$
\begin{gathered}
\gamma_{n}=-<\int \mathbf{J}_{h} \cdot \mathbf{E}_{n} d^{3} \mathbf{r}>/\left(\omega_{n}^{2} A_{n}^{2}\right) \\
\dot{\alpha}_{n}(t)=-<\int \mathbf{J}_{h} \cdot \frac{1}{\omega_{n}} \frac{\partial \mathbf{E}_{n}}{\partial t} d^{3} \mathbf{r}>/ \omega_{n}^{2} A_{n}^{2}
\end{gathered}
$$

where the growth rate $\gamma_{n}=\dot{A}_{n} / A_{n}$ and $<>$ represents averaging over a wave period, and $J_{h}$ is the fast ion current density. The $J_{h}$ is calculated from the fast ion distribution which is obtained by solving the drift-kinetic equation using the $\delta f$ method. A Hamiltonian guiding center code [21] ORBIT is used to follow the particle trajectories in the presence of TAE modes. Notice that Eq. 2 just expresses the energy conservation of the system. Previously, the energy conservation was used to advance the wave amplitude for a single mode with fixed phase. It was observed[22] that resonant particles, which lose energy to the mode through inverse Landau damping, shift outwards, thus the density gradient which gives the free energy to the wave is flattened at the resonant surface as time evolves, eventually leading to mode saturation. These observations are confirmed by using Eq. (2) and (3)directly. We also found that using Eq. (2) and (3) instead of explicit energy conservation can lower the noise significantly. We now consider the simultaneous evolution of multiple modes. Of particular concern in multiple modes case is whether the TAE amplitudes becomes large enough to cause resonance overlap, which can lead to global particle diffusion and energy loss. As shown previously[23], resonance overlap is greatly facilitated by the simultaneous excitation of multiple modes. In the following simulation we use ITER-like parameters: $R=800 \mathrm{~cm}, a=300 \mathrm{~cm}, B=6 T, \delta r=a / 3$. Only $n=2$ and $n=3$ are considered and mode frequencies are $\omega_{n=3} / \Omega_{c}=$ 
$1.34 \times 10^{-3}, \omega_{n=2} / \Omega_{c}=1.12 \times 10^{-3}$ where $\Omega_{c}$ is the on-axis gyro-frequency. The equilibrium particle distribution is assumed to be $f_{0}(r, \varepsilon)=e^{-r^{2} / \delta r^{2}} \varepsilon^{-\frac{3}{2}}$. The mode structures are obtained from NOVA-K code. Figure 4 shows the saturated amplitude of a $n=3$ mode as a function of $\beta_{h}$ from a single mode case and a two mode simulation $(n=2,3)$. We see that for the single mode case the saturation level $A^{1 / 2}$ is approximately linear in $\beta_{h}$, which gives the expected trapping scaling of $A \propto \gamma_{h}$ since the linear growth rate $\gamma_{h}$ is proportional to $\beta_{h}$. Apparent deviation between the saturation levels starts at about $\beta_{h}=0.16 \%$, when resonance overlap occurs. This result shows that the saturation level is enhanced by resonance overlap.

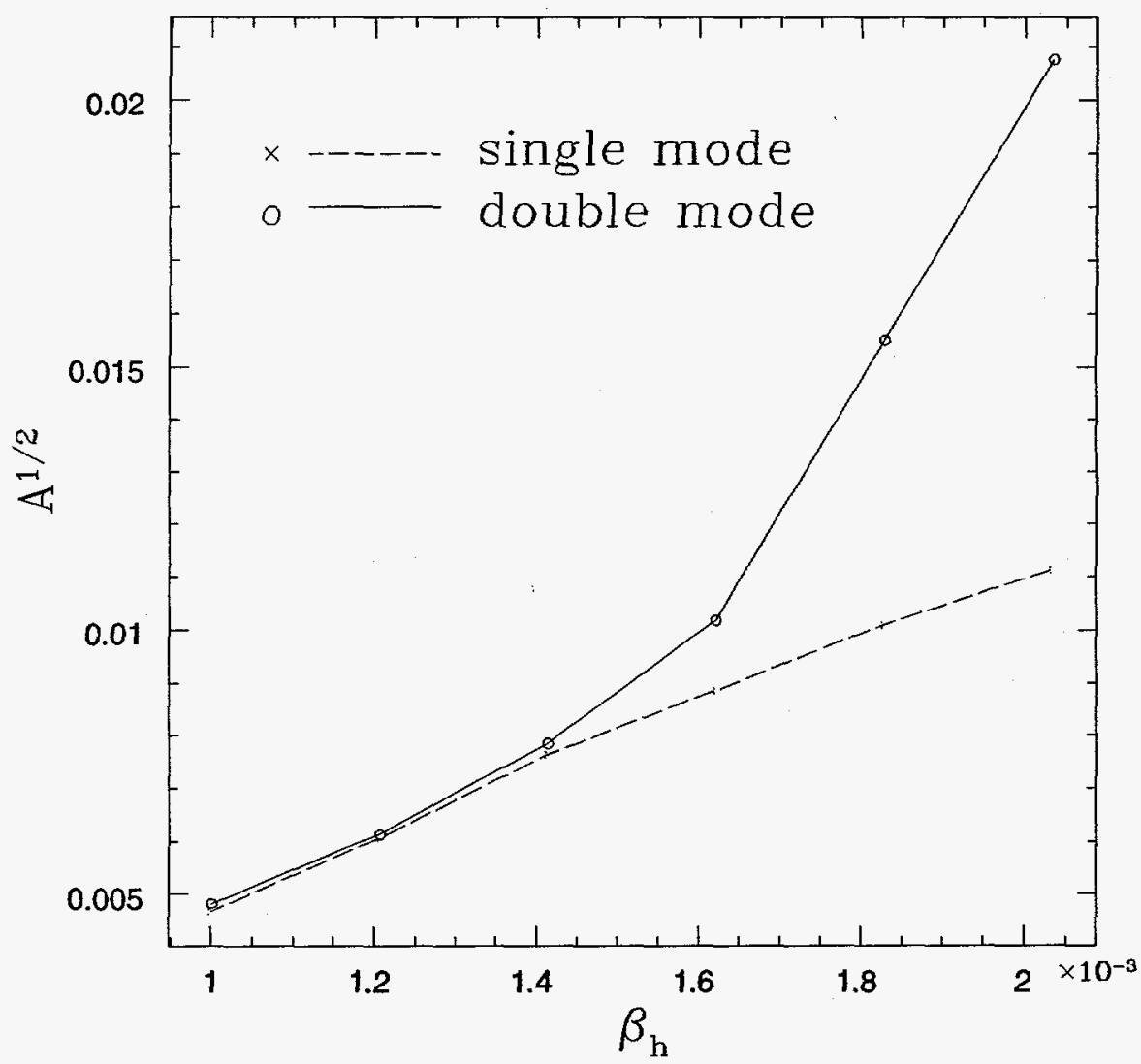

Figure 5: The saturation level as function of $\beta_{h}$ for a single mode case and a double mode case.

In the fully nonlinear hybrid model, the MHD equations are coupled with gyrokinetic energetic particles through the pressure tensor.[20, 24] The plasma is divided into two parts: the bulk plasma, which contains the thermal electrons and ions, and the energetic hot ions. The bulk plasma is described by the ideal MHD equations, whereas the hot ions are described by the gyrokinetic equations[25]. The effects of hot ions couple to the bulk plasma motion through the pressure tensor term in the momentum equation as follows:

$$
\rho_{b} \frac{d \mathbf{v}_{b}}{d t}=-\nabla P_{b}-\left(\nabla \cdot P_{h}\right)_{\perp}+\mathbf{J} \times \mathbf{B}
$$

where the subscript $b$ denotes the bulk part, the subscript $h$ denotes the hot ion component, $P_{b}$ is the isotropic pressure of the bulk plasma, and the $P_{h}$ is 
the pressure tensor of the hot ions. The $P_{h}$ is calculated from the hot ion distribution function $f$ represented by an ensemble of particles which follow the gyrokinetic equations with the self-consistent electromagnetic field. Equation 4 and the other MHD equations are advanced in time using $P_{h}$ given by the particles. The new $\mathbf{E}$ and $\mathbf{B}$ are in turn used to advance the particle quantities in time. The model is fully self-consistent, including self-consistent effects of hot particles on the MHD dynamics and the nonlinear MHD mode coupling.

Using the particle/MHD hybrid $M H 3 D-K$ code, we had found that wave particle trapping is the dominant mechanism for the TAE saturation[24]. In that work, the "double trajectory method" was used to reduce the simulation noise in the linear regime. Two sets of particles are used, one following the equilibrium field and the other the total electromagnetic field. The hot particle pressure tensor $P_{h}(t)$ is replaced by $\left(P_{h}(t)-P_{h, 0}(t)\right)+P_{h, 0}(0)$ where $P_{h, 0}$ is evaluated from the equilibrium orbits. The advantage of the double trajectory method is that it can be applied to any $3 \mathrm{D}$ equilibrium with an arbitrary distribution of particles including a delta function in velocity space. The disadvantage is that it is only valid for the linear regime. In this work, we adopt another noise reduction scheme, namely, the $\delta f \operatorname{method}[26]$, which is valid in both linear and nonlinear regimes. However, it is difficult to apply the scheme in a self-consistent manner to a 3D electromagnetic problem with a self-consistent equilibrium. For the first time, a scheme for the $\delta f$ method is developed for such cases, and implemented in MH3D-K code. The new linear results agree closely with the double trajectory method results, while improved nonlinear saturation results have been obtained for realistic parameters and profiles. The two main components for the self-consistent treatment of the $\delta f$ method are the loading of particles and finding a self consistent equilibrium. The particles are loaded according to an analytic function $f\left(P_{\phi}, E, \mu\right)$. (The equilibrium distribution $f$ is written in terms of the constants of motion in the equilibrium fields, namely, the toroidal angular momentum $P_{\phi}$, the kinetic energy $E$, and the magnetic moment $\mu$.) Specifically, the distribution function is integrated in velocity space to get the particle density, $\rho_{p}(r, \theta)$. Then, the particles are loaded in real space according to $\rho_{p}(r, \theta)$ and in velocity space according to $f_{0}$. Note that the real space variables and the velocity variables are coupled together due to $P_{\phi}$ so that the loading in velocity space can not be separated from that in real space. An equilibrium solution consistent with the analytic distribution $f_{0}\left(P_{\phi}, E, \mu\right)$ is obtained by an iterative method. The particles are first loaded according to an analytic distribution for the initial equilibrium field, and the equilibrium is allowed to relax with the self-consistent pressure. Then, the particles are re-loaded for the new equilibrium field and the equilibrium relaxes again. This process is repeated until the converged equilibrium is consistent with the particle distribution.

In the fully nonlinear simulations, we considered paramèters and profiles similar to those of the TFTR NBI experiments[3] where the TAE modes were destabilized by energetic beam ions: the aspect ratio $R / a=3.0, q(0)=1.0$, $q(a)=3.5$, the plasma density profile $\rho=\rho_{0}\left(1-0.8(r / a)^{2}\right)$, and the hot ion beta $\beta_{h}(0)=2.5 \%$. Figure 6 shows the evolution of the $n=2$ TAE mode for a single mode simulation and a multiple mode simulation with $n=0,1,2$ and 3. We find that the saturation level obtained from a single mode is nearly the same as that from a multiple mode simulation. Thus, the nonlinear mode- 


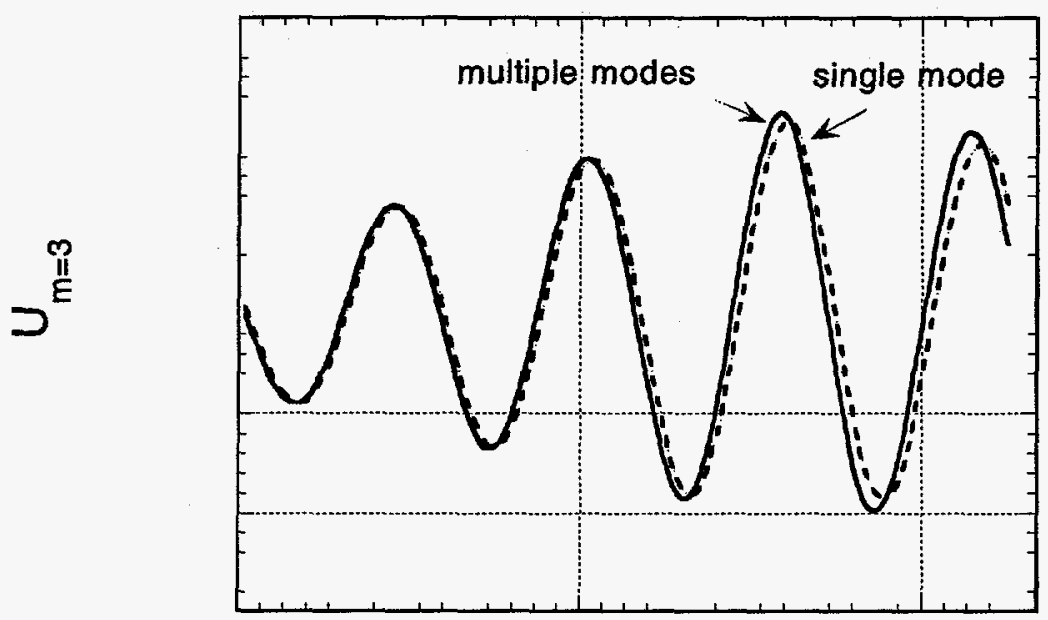

$t$

Figure 6: The mode amplitude versus time obtained with a simple mode (dashed line) and multiple modes (solid line).

mode coupling is not important for the mode saturation, as least for the case considered. Further work is in progress to assess the importance of mode-mode coupling in a wider parameter range.

\section{Conclusions}

The linear stability analysis of TAE modes has been carried out for the parameters and profiles of several tokamak experiments, including the most recent TFTR DT experiments where alpha-driven TAE modes were observed for the first time. The predicted stability trend by the NOVA-K code is well correlated with the observations. However, more work is needed in order to have accurate quantitative comparisons with the experimental thresholds for the instability, especially at levels of individual drive and damping mechanisms. This can be done in two ways. First, the theoretical model can still be improved in order to have all the important damping mechanisms and fast ion drive more selfconsistently. Second, future experiments should be designed to investigate more systematically the dependence of stability on parameter and profiles. Our analysis has shown that the stability is sensitive to plasma profiles, especially the $q$ profile. In the nonlinear regime, hybrid simulations using a quasilinear model as well as a fully nonlinear model have been carried out to assess the nonlinear saturation mechanisms for realistic parameters and profiles. The nonlinear resonance overlapping which enhances the saturation level is demonstrated for ITER parameters. A fully self-consistent $\delta$ f method for the global electromagnetic Particle/MHD model is developed for the first time and is used in the MH3D-K code simulations for realistic parameters. More work is needed to compare the calculated saturated mode amplitudes and related fast ion transport with the experimental observations.

\section{Acknowledgements}


This work was supported by the United States Department of Energy under Contract DE-AC02-76-CHO-3073 and Grant DE-FG02-86ER53223

\section{References}

[1] C. Z. Cheng, L. Chen, and M. S. Chance, Ann. Phys. (N. Y.) 161, 21 (1985).

[2] G. Y. Fu and J. W. Van Dam, Phys. Fluids B1, 1949 (1989).

[3] K. L. Wong et al., Phys. Fluids B4, 2122 (1992).

[4] W. W. Heidbrink et al., Nucl. Fusion 31, 1635 (1991).

[5] J. R. Wilson et al., 1994 IAEA Conf., paper IAEA-CN-56/E-2-2.

[6] M. Saigusa et al., Plasma Phys. Contr. Fusion 37, 295 (1995).

[7] R. Nazikian et al., in this conference, IAEA-CN-64/A2-4.

[8] C. Z. Cheng, Phys. Reports 211, 1 (1992).

[9] G. Y. Fu, C. Z. Cheng and K. L. Wong, Phys. Fluids B5, 4040 (1993).

[10] R. Budny et al., Nucl. Fusion 34, 1247 (1994).

[11] G. Y. Fu et al., Phys. Rev. Lett., 75, 2336 (1995).

[12] G. Y. Fu et al., to appear in Phys. Plasmas.

[13] J. D. Strachan et al., Phys. Rev. Lett., 72, 3526 (1994).

[14] G. Y. Fu, Phys. Plasmas 2, 1029 (1995).

[15] H. L. Berk et al., Phys. Plasmas 2, 3401 (1995).

[16] D. A. Spong et al., 1994 IAEA Conf., Vol. 3, p. 567 (1996).

[17] G. Y. Fu et al., to appear in Nucl. Fusion.

[18] H. Kimura et al., this conference, IAEA-CN-64/E-6.

[19] Y. Chen and R. White, PPPL report 3182, to be published.

[20] W. Park et al., Phys. Fluids B 4, 2033 (1992).

[21] R. White and M. Chance, Phys. Fluids 27, 2455 (1984).

[22] Y. Wu, R. B. White, Y. Chen, and M. N. Rosenbluth, Phys. Plasmas 2, 4555 (1995).

[23] H. L. Berk, B. N. Breizman and H. Ye, Phys. Rev. Lett. 68, 3563 (1992).

[24] G. Y. Fu and W. Park, Phys. Rev. Lett. 741594 (1995).

[25] W. W. Lee, J. Comput. Phys. 72, 243 (1987)

[26] S. E. Parker and W. W. Lee, Phys. Fluids B 5, 77 (1993). 